\title{
Mechanics of Cooling Liquids by Forced Evaporation in Bubbles
}

\author{
Michiel A.J. van Limbeek, ${ }^{1,2,{ }^{*}}$ D. van Buuren, ${ }^{1}$ M.R.P. van den Broek, ${ }^{1}$ H.J.M. ter Brake, ${ }^{1}$ and \\ S. Vanapalli ${ }^{1}$ \\ ${ }^{1}$ Energy Materials and Systems Group, Department of Science and Technology, University of Twente, 7500 AE \\ Enschede, The Netherlands \\ ${ }^{2}$ Physics of Fluids Group, Max Planck Center for Complex Fluid Dynamics, J. M. Burgers Center for Fluid \\ Dynamics and MESA + Research Institute, Department of Science and Technology, University of Twente, P.O. Box \\ 217, 7500 AE Enschede, The Netherlands
}

(Received 18 July 2018; revised manuscript received 15 January 2019; published 14 May 2019)

\begin{abstract}
The injection of a nondissolvable gas into a saturated liquid results in subcooling of the liquid due to forced evaporation into the bubble. Previous studies have assumed the rate of evaporation of liquid into the bubble to be independent of the degree of subcooling. In our study, we quantify the bubble growth by direct observation using high-speed imaging and prove that this hypothesis is not true. A phenomenological model of the bubble growth as a function of the degree of subcooling is developed and we find excellent agreement between the measurements and theory. This bubble-cooling process is employed in cooling a liquid. By identification of all heat flows, we can describe the cool-down curve well using bubble cooling, which provides an alternative cooling method for liquids without the use of complicated techniques.
\end{abstract}

DOI: 10.1103/PhysRevApplied.11.054038

\section{INTRODUCTION}

The evaporation of liquids is an efficient way to transfer heat away from a hot solid, a method frequently employed in the context of heat pipes, power plants, and industrial spray cooling. An everyday example is the use of transpiration to regulate our body temperature. The amount of liquid that can evaporate into vapor depends largely on the liquid's temperature and its vapor pressure. When injecting a bubble of a noncondensable substance into a liquid bath, this partial vapor pressure is initially zero, resulting in immediate evaporation and, hence, cooling of the liquid. In contrast to the energy required for expanding a bubble through a hydrostatic gradient, the evaporative process studied here requires much more energy and is of more importance for applications. Also, the gas in the bubbles stabilizes condensing bubbles to prevent them from shrinking in subsaturation conditions. Potentially, this alters the characteristics in turbulent flow, which is now mainly studied for pure vapor bubbles [1-6].

The cooling of the liquid bath is often not favorable. In many cases, this is a side effect of another process, which leads to efficiency losses. As an example, in a bubble-column reactor, the injected gas is used to react with the liquid [7-10] or to remove already dissolved gases (sparging) [11-13]. In both systems, the aforementioned evaporative mechanism involves an energy penalty in the form of latent heat. Moreover, in the case of sparging, a

\footnotetext{
*m.a.j.vanlimbeek@utwente.nl
}

fraction of the liquid will also be removed by the bubble process.

For some systems, however, the bubble cooling is employed as a cooling mechanism. For example, cryogenic liquids are often at their saturation temperature following their liquefaction. It has been found that the bubbling of helium gas into a liquid-nitrogen tank lowers the temperature of the liquid by more than $10 \mathrm{~K}$ and suppresses (vapor) bubble generation [14-18]. First, subcooling the liquid allows studies to be performed in cryogenic liquids without any disturbances caused by vapor bubbles [19]. Another advantage is that the cooling rate of quenching procedures is greatly enhanced when the liquid is subcooled, which is of great importance in the context of cryopreservation [20,21]. Furthermore, in contrast to depressurizing a tank to achieve a (temporary) lower saturation temperature and then increasing the pressure again, this bubble-cooling method does not require the system to withstand reduced pressures and it will offer a continuous rather than a batch subcooling of the liquid.

For both cases, whether the cooling is desirable (bubble cooling) or not (bubble columns), little is known about the quantities of energy and mass that are exchanged during this process. Previous attempts [12,13,22] used a global approach by measuring the macroscopic variables, such as mass loss over time and liquid-bath temperature. This, however, gives little insight into the microscopic processes and only yields empirical expressions. In this study, we optically investigate the heat and mass exchange for a cryogenic system, where helium gas is injected into liquid 

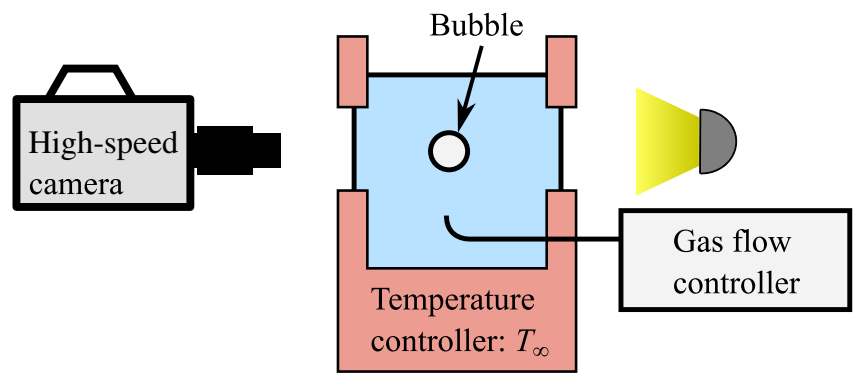

FIG. 1. A schematic of the setup to measure the bubble growth. Most of the components described in the text are omitted for clarity.

nitrogen, and a noncryogenic case in which nitrogen gas is fed into a water bath. We focus on how the bubbles grow by forced evaporation, depending on the temperature of the liquid bath. We propose a theoretical model of this cooling mechanism and verify the model by optical observations. Second, we characterize the cooling of a known system, using forced evaporation, applying our earlier results.

\section{EXPERIMENTAL ASPECTS}

\section{A. Single-bubble observations}

The growth of the bubbles is measured using a setup that, in essence, has only four major components: a temperature-controlled container, a high-speed camera, a light source, and a bubble injector (see Fig. 1). A transparent container is used for optical access and is equipped with a temperature controller. We use a Photron APXRS high-speed camera, operating at 1000 frames $\mathrm{s}^{-1}$ and a shutter speed of $0.5 \mathrm{~ms}$, to prevent motion blurring. A K2 Distamax lens system is used to give a field of view that is roughly $12 \mathrm{~mm}$ wide. A fiber Schott lamp with a diffuser is used as a light source. The gas is injected using an upright needle and controlled by a mass flow controller. The flow rate is in the order of $10^{-2} \mathrm{mg} \mathrm{s}^{-1}$ to ensure sufficient spacing between the bubbles to be able to perform accurate bubble-volume measurements. The density of the gas, required for calculating the volumetric flow rate, is evaluated for all measurements at the bath temperature. Further liquid-specific details are discussed in the Appendix.

\section{B. Cooling-bath setup}

In addition to the optical measurements of the bubble growth, we employ the following method to study the cooling down of a well-defined system (see Fig. 2). A small amount of liquid nitrogen is poured into a steel tube, which is closed at the bottom. This geometry allows accurate calculation of the total heat capacity, for use in further calculations. Bubbles are generated inside this liquid bath and the temperature and liquid level are monitored during the

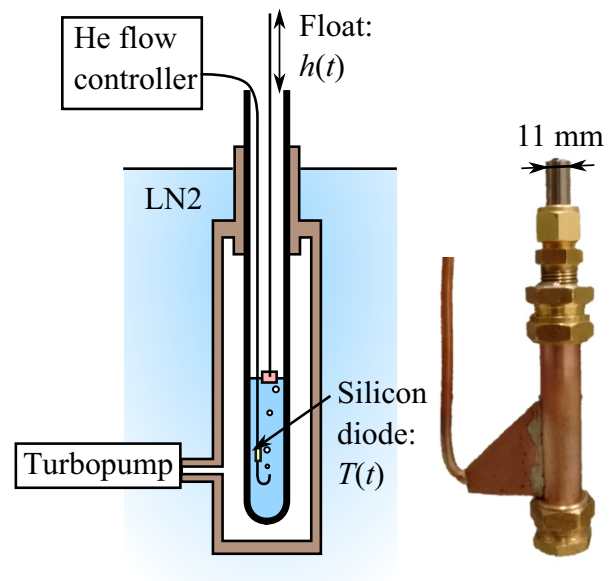

FIG. 2. The experimental setup for the cooling measurements. The schematic on the left is a simplified representation, whereas the picture on the right shows the realized setup without the temperature sensor and tubing for the helium gas. The level of the liquid is measured by a float on which a long glass capillary is placed.

experiment. The liquid level is measured by means of a float, which is equipped with a glass capillary to allow the tracking of its position outside the tube. To prevent heat losses, we create a vacuum around the bottom and the side walls, using a second larger tube. The space created is maintained at a pressure of $10^{-5} \mathrm{~Pa}$. Radiation is prevented by submerging the setup in a large liquid-nitrogen bath, thus maintaining the outer tube and the open top of the steel tube at $T=77 \mathrm{~K}$.

\section{RESULTS AND DISCUSSION}

As mentioned before, measurements are performed on helium-gas bubbles injected in a liquid-nitrogen bath and on nitrogen-gas bubbles cooling a water bath. As an example, recordings for the first system, where the bath temperatures are at $71.2 \mathrm{~K}$ and $76.8 \mathrm{~K}$, are shown in Fig. 3.

For every frame, we obtain the volume of the bubbles assuming axisymmetry. Details of the analysis can be found in the Appendix. The (unfiltered) time series obtained in this way are presented in Fig. 3. Comparing the two, one can readily see that the temperature of the bath is important: with increasing temperature, the bubble frequency is increasing as well as the size of the bubbles, which is also visible in the images of Fig. 3.

The size and frequency difference between the bubbles for different temperatures can be understood by a pressure balance. Let $p_{g}$ be the gas pressure (in this case, of helium) and let $p_{v}$ be the vapor pressure (in this case, of nitrogen). Then, we obtain the following:

$$
p_{g}+p_{v}=p_{\infty}+2 \sigma / R+\rho_{l} g h
$$




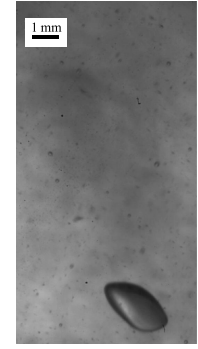

$71.2 \mathrm{~K}$

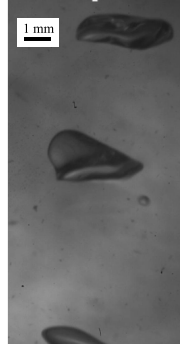

$76.8 \mathrm{~K}$

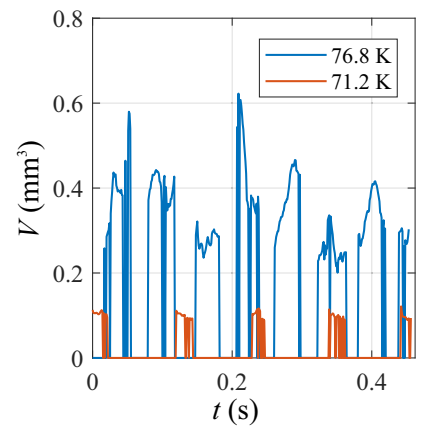

FIG. 3. Snapshots of bubbles in a liquid-nitrogen bath at two different bath temperatures. Helium gas is injected from below (not visible), where the liquid nitrogen evaporates into the bubble, increasing the bubble size. Images are taken far away from the injection point to ensure that the final bubble size is observed. In the figure to the right, each peak represents a unique bubble. Both the bubble frequency as well as the bubble volume increase with increasing bath temperature. The increase in volume allows for larger surface deformations, reflected in fluctuations in the recorded volume.

where $p_{\infty}$ is the ambient pressure of the container. The second and third terms on the right-hand side denote the Laplace pressure due to the surface tension $\sigma$ and the hydrostatic pressure term (using $\rho_{l}$ as the liquid density, $g$ as the gravitational constant, and $h$ as the distance from the free surface of the bath). For millimetric bubbles inside a 0.1-m bath, these two terms are negligible. Hence, Eq. (1) reduces to just $p_{g}+p_{v}=p_{b}$, for which we write $p_{b}=p_{\infty}$ as the bath pressure. This balance assumes the bubble to be at equilibrium with the liquid bath, in terms of heat as well as mass transfer.

We measure the final bubble growth a few centimeters from the injection point, to avoid the complex phenomena involving the dynamics. At this position, the bubble has reached its final size and is at thermal equilibrium [12,23] with the well-mixed bath [6]. Note that the bath is well mixed by the rising bubbles and that recirculation inside the bubble reduces the mixing time scales further. Additional observations reveal the growth that has already taken place while the bubble was still attached to the injection needle.

The gas pressure can be found using the ideal gas law, $p_{g}=N k_{B} T / V$, for the bubble before and after evaporative growth. Here, $N=p_{b} V_{0} /\left(k_{B} T\right)$, the number of molecules in the initial bubble of volume $V_{0}$, from which we obtain, for the gas pressure, $p_{g}=p_{b} V_{0} / V$, where $V$ is the final volume of the gas-vapor bubble. Substitution into the reduced form of Eq. (1) gives the pressure balance with the saturation pressure:

$$
p_{v}=p_{b}\left(1-\frac{V_{0}}{V}\right) \rightarrow \frac{V}{V_{0}}=\left(1-\frac{p_{v}}{p_{b}}\right)^{-1}
$$

The vapor pressure is strongly temperature dependent and can be described well by the Clausius-Clapeyron equation or by the empirical Antoine equation. When using the latter, the growth ratio turns out to be the following:

$$
\frac{V}{V_{0}}=\left(1-\frac{1}{p_{b}} 10^{\left\{A-\left[B /\left(T_{b}+C\right)\right]\right\}}\right)^{-1},
$$

where $A, B$, and $C$ are fluid-specific constants describing the saturation pressure and $T_{b}$ is the bath temperature. The vapor pressure according to the Clausius-Clapeyron equation is as follows: $p_{v}=\exp \left[-L / R_{\text {gas }}\left(1 / T_{b}-1 / T_{r}\right)\right]$, where the subscript $r$ denotes a reference state, $L$ is the molar latent heat of evaporation, and $R_{\text {gas }}$ is the universal gas constant. A further simplification can be obtained by using the saturation conditions of the substance at $p_{r}=p_{b}$ as the reference state, i.e., $T_{r}=T_{\text {sat }}\left(p_{b}\right)$, from now on abbreviated as just $T_{s}$. As a result, the growth ratio can be expressed as follows:

$$
\frac{V}{V_{0}}=\left(1-\exp ^{-L / R_{\mathrm{gas}}\left(1 / T_{b}-1 / T_{s}\right)}\right)^{-1} .
$$

The degree of subcooling, $-\Delta T$, equals $-\left(T_{s}-T_{b}\right)$. We can write $1 / T_{b}=\left(1 / T_{s}\right)+\left(\Delta T / T_{s}^{2}\right)+\mathcal{O}\left(\Delta T^{2}\right)$, where the higher-order terms can be neglected for relatively small $\Delta T$, resulting in

$$
\frac{V}{V_{0}}=\left(1-\exp ^{-\Delta T L /\left(R_{\text {gas }} T_{s}^{2}\right)}\right)^{-1} .
$$

When rescaling all temperatures by $\Delta \tilde{T}=\Delta T L /\left(R_{\text {gas }} T_{s}^{2}\right)$, we can collapse all of the data onto a single curve, as shown in the inset of Fig. 4. This provides a powerful tool to compare the bubble expansion ratios for a given temperature drop between various substances. For example, for a bubble to expand to triple the original volume, we find that $-\Delta \tilde{T}=\ln (1-1 / 3) \approx-0.41$. Table I shows the corresponding temperature drop for the six substances used in Fig. 4, displaying good agreement.

An indirect measure of Eq. (4) is the observed amount of volume rising towards the free surface relative to the amount of gas being injected per unit of time, i.e., $\dot{V}_{f} / \dot{V}_{0}$, in which $f$ and 0 denote the final volume and the injected volume, respectively. $V_{0}$ is obtained from the mass flow rate as set by the flow controller using the density of the gas at the bath temperature, whereas $\dot{V}_{f}$ is obtained by dividing the summed volumes of all individual bubbles passing the camera by the total time of the measurement. In this way, we avoid difficulties in the determination of the initial bubble size, which differs from bubble to bubble. We perform experimental validation of this relation by measuring the ratio in volume flux, using the setup presented in Fig. 1. The results are shown in Fig. 5 for gaseous nitrogen injection into a water bath and gaseous helium injection into a 
TABLE I. The absolute cooling temperature (in kelvin) using the Antoine equation and the rescaled formula for a bubble expanding to three times its original volume (Antoine coefficients taken from [24]).

\begin{tabular}{lcccccc}
\hline \hline & Water & Nitrogen & Ethanol & Oxygen & Isopropanol & Isopentane \\
\hline Eq. (3) & 11.2 & 3.6 & 10.1 & 3.7 & 9.7 & 11.2 \\
$\Delta \tilde{T} R_{\text {gas }} T_{s}^{2} / L$ & 11.7 & 3.7 & 10.1 & 4.1 & 9.8 & 12.2 \\
\hline \hline
\end{tabular}

liquid-nitrogen bath. As can be seen, the results compare well with the predictions of Eq. (4) for both systems.

In a further study, the setup described in Fig. 2 is used to study the time evolution of a liquid volume being cooled down by forced evaporation. We prepare $4.8 \mathrm{~mL}$ of liquid nitrogen and use helium-gas injection to monitor the decrease in temperature $T$ and the liquid level $h$. The results are presented in Fig. 6, where at $t=225 \mathrm{~s}$ helium gas is injected at a rate of $2 \times 10^{-1} \mathrm{mg} \mathrm{s}^{-1}$. An immediate decrease in temperature can be observed, as well as a decrease in nitrogen level as a result of the forced evaporation. The decrease in temperature of the liquid can be estimated by considering the power taken from the liquid (and the container). The power can be expressed as $L \rho_{b}\left(d V_{b} / d t\right)$, which is equivalent to $L \rho_{v}(d V / d t)$. The bubble expansion rate can be expressed as $\left(d V_{0} / d t\right)(V-$ $\left.V_{0}\right) / V_{0}$, where $d V_{0} / d t$ is the volumetric flow of the helium gas $\dot{V}_{0}$. As a result, the rate at which the bath temperature decreases is estimated as $d T / d t \approx \dot{V}_{0} L\left[\left(V / V_{0}\right)-1\right] \rho_{v} / C$, where $C$ is the total heat capacity of the liquid nitrogen and the steel tube surrounding it. With $\dot{V}_{0}=3.2 \times$ $10^{-7} \mathrm{~m}^{3} \mathrm{~s}^{-1}, L=2 \times 10^{5} \mathrm{~J} \mathrm{~K}^{-1}, \rho_{v}=4.6 \mathrm{~kg} \mathrm{~m}^{-3}$, and $C=7.8 \mathrm{~J} \mathrm{~K}^{-1}$, we find $\Delta T / \Delta t \approx 10^{-1} \mathrm{~K} \mathrm{~s}^{-1}$, which is the right order of magnitude for the initial cooling-down rate, as can be seen from Fig. 6 .

Applying the established theory, we can use the temperature data to calculate the expected decrease in the nitrogen level and compare this with the direct measurements of the float (see Fig. 6). It can be seen that the model predicts

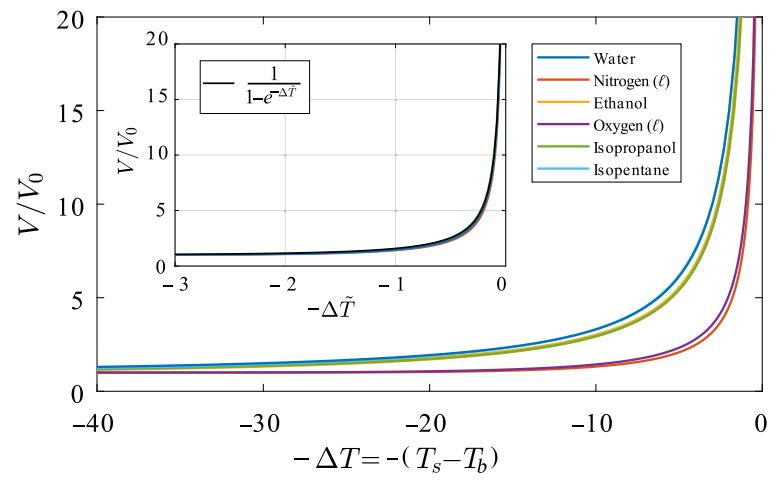

FIG. 4. The bubble growth ratio $V / V_{0}$ for different liquids, based on the Antoine equation, with constants $A, B$, and $C$ taken from [24]. Using the rescaled temperature $\Delta \tilde{T}=\Delta T L /\left(R_{\text {gas }} T_{s}^{2}\right)$, one can collapse all of the liquids onto a single curve, as shown in the inset. the evaporation quite well, whereas for low temperatures an overprediction is found. The system seems to stabilize at a temperature of about $73.5 \mathrm{~K}$, at which the liquid level also hardly decreases. We attribute this to recondensation of nitrogen onto the tube wall above the liquid bath, by the following mechanism. As a result of the top part of the tube being in contact with the liquid-nitrogen bath, it will be at $78 \mathrm{~K}$, while the lower part will be at the temperature of the subcooled liquid. Therefore, a thermal gradient emerges in the few centimeters between the two parts (see
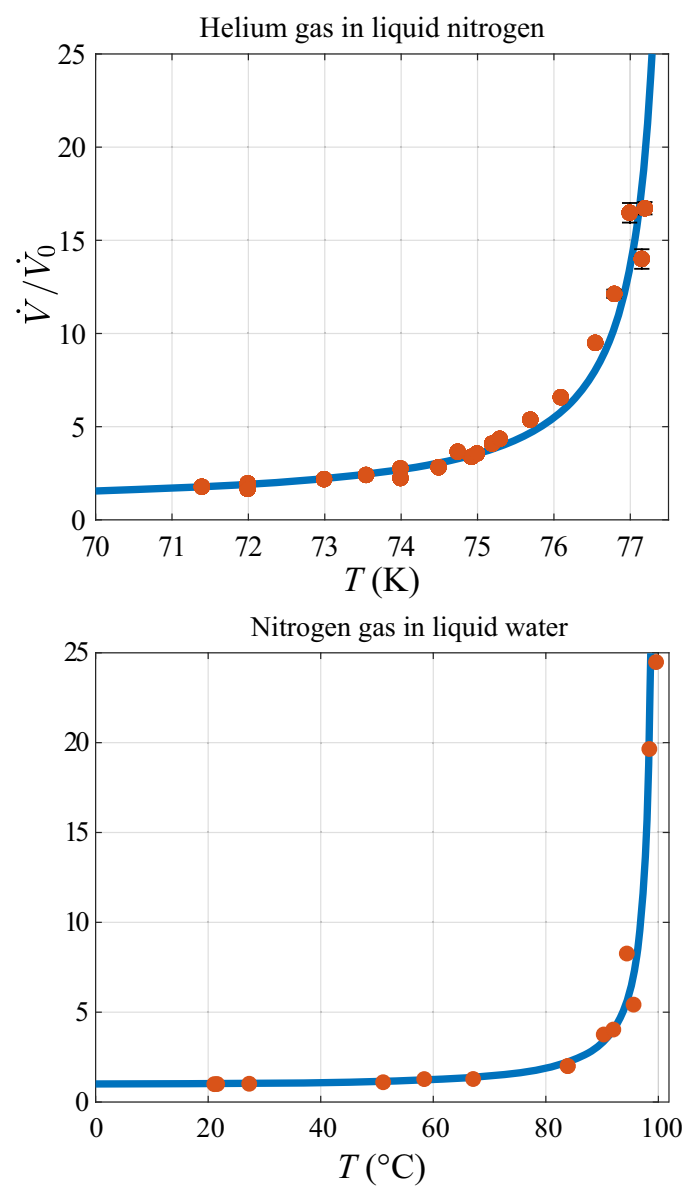

FIG. 5. Measurements of the ratio between the injected gas volume $\dot{V}_{0}$ and the (optically) observed bubble volume $\dot{V}$ passing per unit time. Two systems are studied here: helium-gas injection into liquid nitrogen (top) and nitrogen gas into a water bath. Both measurements show good agreement with Eq. (3) (solid line) for all temperatures. Error bars smaller than the corresponding data points are omitted. 

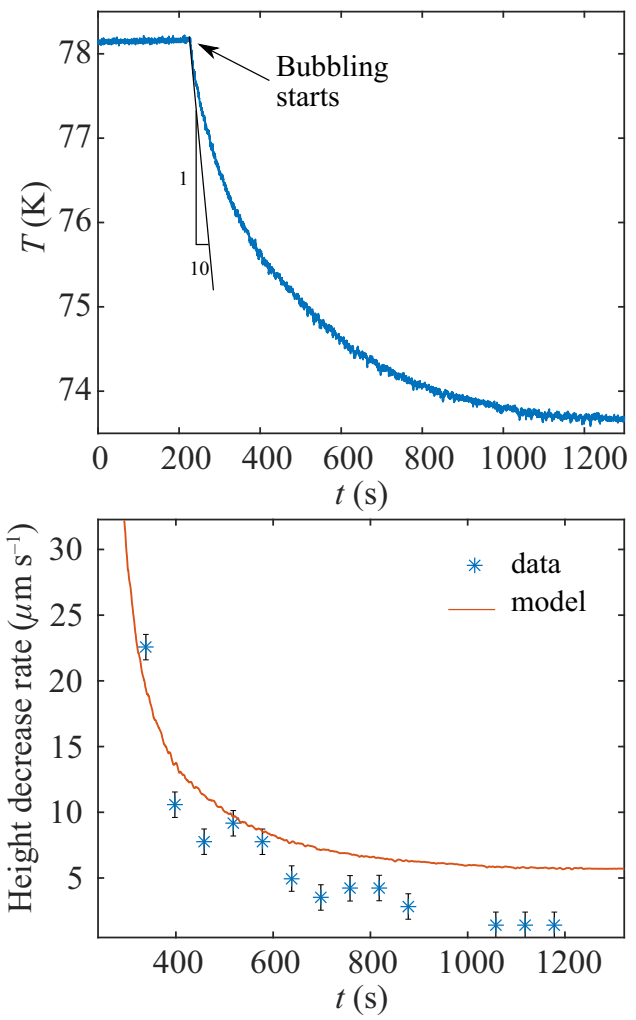

FIG. 6. The bubble experiment consisting of helium gas in a bath of $4.8 \mathrm{~mL}$ of liquid nitrogen. The top panel shows the temperature decrease as a result of the forced evaporation, where an initial slope $\Delta T / \Delta t \approx 10^{-1} \mathrm{~K} \mathrm{~s}^{-1}$ is found when the bubbling starts. The bottom panel shows the decrease in liquid height, as measured by the float.

Fig. 2). The mixed content of the bubbles has a lower density than that of pure nitrogen vapor; thus it flows out of the tube by natural convection, while pure nitrogen gas at $78 \mathrm{~K}$ sinks into the tube. The heat of condensation will compensate the heat of evaporation taken from the liquid through the injection of the helium gas, negating the vapor-cooling method.

In terms of cooling power per injected gas volume, we find that the bubble-cooling method excels close to the saturation temperature of the liquid, made visible by the rapid decrease in bath level when the bubbling has just started. This is in agreement with the visual measurements of Fig. 5, as can be seen by the asymptotic behavior of both the measurements and the theory. As a result of our study, the efficiency of a cooler or the mass loss in a bubble column is therefore at a maximal close to the saturation temperature of the bath.

\section{CONCLUSION}

We study the cooling behavior of a liquid by gas injection. As a result of the initial partial pressure being zero, the liquid evaporates into the gas bubble until equilibrium is reached. The evaporation process takes energy from the bath, resulting in a decrease of the bath temperature, as observed in our experiments. The optical measurements of the bubble growth in two different liquid-bubble pairs show excellent agreement with our theoretical description of the phenomena. Our proposed rescaling of the temperature using liquid-specific parameters allows for easy comparison and understanding of this phenomenon. It allows the heat and mass losses in the context of chemical reactors to be indicated, while predicting the efficiency for future applications using this technique as a cooling method.

\section{APPENDIX}

\section{Additional experimental methods}

For the study of water, the container is made of glass and is placed inside a temperature-controlled copper block. Using a flow controller, the nitrogen flow is set to be $\dot{m}_{0}=$ $5.2 \times 10^{-2} \mathrm{mg} \mathrm{s}^{-1}$.

The bubble growth in liquid nitrogen is studied by helium-gas injection at a flow rate of $\dot{m}_{0}=2.0 \times$ $10^{-2} \mathrm{mg} \mathrm{s}^{-1}$. In order to study liquid nitrogen, it is of great importance to limit the heat losses for the setup. Therefore, the container is placed in a sacrificial bath, which is also filled with liquid nitrogen. The gas flow is precooled by leading the tubing through this bath. The whole setup is put inside a cryostat, access to which is only at the top, to prevent heat losses. As a consequence, the optical measurements are performed from the top, using mirrors for both the camera and the light source. An evaporator is put inside the setup to generate cold nitrogen gas to prevent icing of the setup, which otherwise would hinder optical access. Since the primary flow lacks the cooling capacity to cool the setup down, a large, secondary helium-gas flow is used to cool down the bath by the same mechanism of bubble cooling.

\section{Image processing}

The images from the camera are analysed using automated image processing. First, edge detection is used, by means of a "Canny" filtering technique [25]. The resulting binary image contains various regions. When choosing the proper filtering parameters, the largest of these regions are the bubbles for which the position and volume has to be calculated. The volume is obtained by first slicing the area into horizontal lines, from which the center position $r_{c}(z)$ and the radius $R(z)$ can be determined. Assuming axisymmetry, this allows us to express the bubble volume as $V=2 \pi \int_{0}^{h}\left[R(z)-r_{c}(z)\right]^{2} d z$, which is implemented numerically using the trapezoid integration method. 


\section{Postprocessing}

Since the bubble has a size of roughly the capillary length $\left(\sigma / \rho_{1} g\right)^{1 / 2}$, surface deformations are expected. This effect is amplified by the increasing velocity and size of the bubble during the rise toward the free surface of the bath. Therefore, the bubble is no longer axisymmetric and the apparent volume fluctuates between frames. Temporal averaging is therefore performed for every bubble to obtain a more accurate bubble volume.

\section{Error analysis}

Multiple systematic errors can be identified, which are found to be negligible: the calibration error of the camera and uncertainties in the temperature probe and the gas flow controller. The largest error in our measurements, however, emerges from the aforementioned bubble shape fluctuations. Therefore, we use the variance in measured bubble size as a statistical error: $\sigma^{2}=\Sigma_{j} \sigma_{j}^{2}$, where $\sigma_{j}^{2}$ indicates the variance for every bubble ${ }_{j}$. The second source of error originates from the uncertainty of the temperature measurements, which is $\pm 0.2 \mathrm{~K}$.

[1] N. Zuber, On the stability of boiling heat transfer, Trans. Am. Soc. Mech. Engrs. 80 (1958).

[2] V. Dhir, Boiling heat transfer, Ann. Rev. Fluid Mech. 30, 365 (1998).

[3] R. Lakkaraju, L. E. Schmidt, P. Oresta, F. Toschi, R. Verzicco, D. Lohse, and A. Prosperetti, Effect of vapor bubbles on velocity fluctuations and dissipation rates in bubbly Rayleigh-Bénard convection, Phys. Rev. E 84, 036312 (2011).

[4] L. E. Schmidt, P. Oresta, F. Toschi, R. Verzicco, D. Lohse, and A. Prosperetti, Modification of turbulence in RayleighBénard convection by phase change, New J. Phys. 13, 025002 (2011).

[5] L. Biferale, P. Perlekar, M. Sbragaglia, and F. Toschi, Convection in Multiphase Fluid Flows Using Lattice Boltzmann Methods, Phys. Rev. Lett. 108, 104502 (2012).

[6] B. Gvozdić, E. Alméras, V. Mathai, X. Zhu, D. P. van Gils, R. Verzicco, S. G. Huisman, C. Sun, and D. Lohse, Experimental investigation of heat transport in homogeneous bubbly flow, J. Fluid Mech. 845, 226 (2018).

[7] K. Akita and F. Yoshida, Bubble size, interfacial area, and liquid-phase mass transfer coefficient in bubble columns, Ind. Eng. Chem. Process Des. Dev. 13, 84 (1974).
[8] W.-D. Deckwer, On the mechanism of heat transfer in bubble column reactors, Chem. Eng. Sci. 35, 1341 (1980).

[9] S. C. Saxena, Bubble column reactors and Fischer-Tropsch synthesis, Catal. Rev. 37, 227 (1995).

[10] K. Shimizu, S. Takada, K. Minekawa, and Y. Kawase, Phenomenological model for bubble column reactors: Prediction of gas hold-ups and volumetric mass transfer coefficients, Chem. Eng. J. 78, 21 (2000).

[11] J. N. Brown, M. Hewins, J. H. Van Der Linden, and R. J. Lynch, Solvent degassing and other factors affecting liquid chromatographic detector stability, J. Chromatogr. A 204, 115 (1981).

[12] E. W. Tow and J. H. Lienhard, Experiments and modeling of bubble column dehumidifier performance, Int. J. Therm. Sci. 80, 65 (2014).

[13] M. Shahid, C. Fan, and R. M. Pashley, Insight into the bubble column evaporator and its applications, Int. Rev. Phys. Chem. 35, 143 (2016).

[14] A. K. Stober, G. J. Minkoff, and F. I. Scherbert, Suppression of bubbling in boiling refrigerants, Nature 180, 1413 (1957).

[15] A. Schmidt, Experimental investigation of liquid-hydrogen cooling by helium gas injection, Adv. Cryog. Eng. 8 (1963).

[16] F. W. Lytle and J. T. Stoner, Cryogenic cooling by noncondensible-gas injection, Science 148, 1721 (1965).

[17] J. Xu, H. J. Jänsch, and J. T. Yates, Jr., Cryogenic trick for enhanced cooling using liquid nitrogen, J. Vac. Sci. Technol. A: Vac. Surfaces Films 11, 726 (1993).

[18] S. Takayoshi, W. Kokuyama, and H. Fukuyama, The boiling suppression of liquid nitrogen, Cryogenics 49, 221 (2009).

[19] A. Townsend and R. Mishra, Design and development of a helium injection system to improve external leakage detection during liquid nitrogen immersion tests, Cryogenics 79, 17 (2016).

[20] R. Leek, in Breast Cancer Research Protocols (Humana Press, 2006), p. 25-28.

[21] M. M. Morente, R. Mager, S. Alonso, F. Pezzella, A. Spatz, K. Knox, D. Kerr, W. N. M. Dinjens, J. Oosterhuis, K. H. Lam et al., Tubafrost 2: Standardising tissue collection and quality control procedures for a European virtual frozen tissue bank network, Eur. J. Cancer 42, 2684 (2006).

[22] P. Saha and P. Sandilya, A dynamic lumped parameter model of injection cooling system for liquid subcooling, Int. J. Therm. Sci. 132, 552 (2018).

[23] R. Clift, J. R. Grace, and M. E. Weber, Bubbles, Drops, and Particles (Courier Corporation, 2005).

[24] NIST webbook http://wtt-pro.nist.gov/wtt-pro/.

[25] J. Canny, A computational approach to edge detection, IEEE Trans. Pattern Anal. Machine Intell. 8, 679 (1986). 